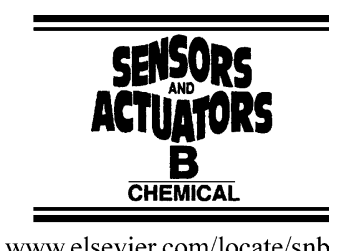

\title{
Performance of a micro-engineered ultrasonic particle manipulator
}

\author{
N.R. Harris ${ }^{\mathrm{a}, *}$, M. Hill ${ }^{\mathrm{b}}$, R. Townsend ${ }^{\mathrm{b}}$, N.M. White ${ }^{\mathrm{a}}$, S.P. Beeby ${ }^{\mathrm{a}}$ \\ ${ }^{a}$ ECS, University of Southampton, ESD Group, Department of Electronics and Computer Science, Mountbatten Building, \\ Highfield, Southampton SO17 1BJ, UK \\ b SES, University of Southampton, Highfield, Southampton SO17 1BJ, UK
}

Available online 25 April 2005

\begin{abstract}
(C) 2005 Elsevier B.V. All rights reserved.

Keywords: Microfluidic; Ultrasonic; Separator

\section{Introduction}

High-frequency acoustic standing waves can be used to separate materials with different acoustic characteristics. The technique can be used to agglomerate particles, to hold particles within a flow, or to manipulate particles within the flow [1].

This paper briefly describes the construction and then describes both the acoustic modelling and the particle trajectory modelling of an ultrasonic device that uses this principle to separate particles. The combination of these models allows prediction of the performance of the separator, and these predicted results are compared with experimental performance.
\end{abstract}

An ultrasonic microfluidic particle manipulator has been modeled and its experimentally measured separation performance has been compared with the modeled results for $1 \mu \mathrm{m}$ latex particles, and yeast particles in water.

\section{Construction and modelling}

The device has been constructed in Pyrex and silicon, to make use of standard silicon processes. It consists of a silicon substrate with channels etched through it, and a Pyrex top that has a cavity etched into it, as shown schematically in Fig. 1.

The depth of the cavity is $175 \mu \mathrm{m}$, and the unetched thickness of the Pyrex wafer is $1.7 \mathrm{~mm}$. The silicon is $525 \mu \mathrm{m}$

\footnotetext{
* Corresponding author.

E-mail address: nrh@ecs.soton.ac.uk (N.R. Harris).
}

thick. These dimensions have been chosen as a result of a modelling study [3] and extended in [2].

These studies predicted a half-wave mode at $3.5 \mathrm{MHz}$ and this was confirmed in [2], and this was the mode used for the results described here.

If the PZT transducer is driven at the required resonant frequency of the chamber, the result is an acoustic standing wave generated in the cavity. Particles within the fluid then move towards the pressure nodal plane, driven by acoustic radiation forces. In this case, the device is operated in half-wave mode and so the nodal position is in the centre of the cavity. The device has two outlets, and by selectively adjusting the relative outputs of these two channels, it is possible to arrange to have one flow predominately made up of the cleaner fluid, and the other to contain the majority of the particles. However, to make useful predictions about the separation performance of such a device, it is necessary to incorporate information about how specific particles behave under the influence of the acoustic field. In many ways this interaction between the acoustic field and the hydrodynamic system is the most important part of the system to model, and this model has been used to make predictions of the separation performance of the device as the transducer drive voltage is altered for a given flow rate, for a range of different particles and these predictions are compared with experimental results in this paper. 


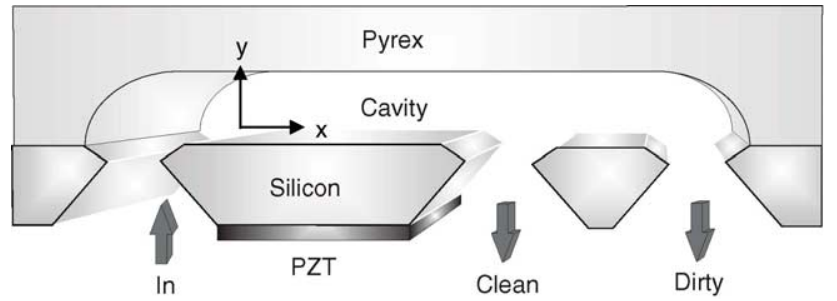

Fig. 1. Schematic of the separator device.

\subsection{Predicted performance}

As a particle passes along the main channel of the separator, it is subject to acoustic radiation, drag and buoyancy forces. Less significant forces such as those due to lateral acoustic effects, acoustic streaming and interparticle forces [1] are not considered by the following treatment. The dominant forces mentioned are solved numerically within MAT$\mathrm{LAB}$, resulting in a description of the particle trajectory as the particle passes through the ultrasonic separator. A series of particle trajectories are used to determine the relative spacing of particles and therefore the concentration as the particles move along the channel. This is a numerical method which enables both frequency dependence and non-uniform flow to be easily considered.

\subsubsection{Acoustic radiation force}

Within an acoustic field, the time averaged acoustic force on a particle, $F_{\text {ac }}$, in a direction normal to the transducer is given by [1]:

$$
\left\langle F_{\mathrm{ac}}(x, y, z)\right\rangle=-\nabla\left\langle\phi^{\mathrm{G}}(x, y, z)\right\rangle
$$

with

$$
\begin{aligned}
& \left\langle\phi^{\mathrm{G}}(x, y, z)\right\rangle \\
& \quad=-V\left[\frac{3\left(\rho_{\mathrm{p}}-\rho_{\mathrm{f}}\right)}{2 \rho_{\mathrm{p}}+\rho_{\mathrm{f}}}\left\langle\bar{E}_{\mathrm{kin}}\right\rangle-\left(1-\frac{c_{\mathrm{f}}^{2} \rho_{\mathrm{f}}}{c_{\mathrm{p}}^{2} \rho_{\mathrm{p}}}\right)\left\langle\bar{E}_{\mathrm{pot}}\right\rangle\right]
\end{aligned}
$$

where $V$ is the particle volume, $\rho_{\mathrm{p}}$ and $\rho_{\mathrm{f}}$ the particle and fluid densities, respectively, $c_{\mathrm{p}}$ and $c_{\mathrm{f}}$ the speed of sound for the particle and fluid mediums, respectively, $\left\langle\bar{E}_{\text {kin }}\right\rangle$ and $\left\langle\bar{E}_{\text {pot }}\right\rangle$ the time-averaged kinetic and potential energies at a point in the field, respectively and $\phi^{\mathrm{G}}$ the gradient of radiation force potential. The orientation of the $x$ and $y$ axes are indicated in Fig. 1.

$F_{\text {ac }}$ can easily be determined for the resonant case and where the nodal position and level of acoustic energy within the channel is known [1]. However, by using an acoustic impedance transfer model $[7,8]$, the variation in acoustic velocity and pressure through the device can be determined, which allows the kinetic and potential energies, $\left\langle\bar{E}_{\text {kin }}\right\rangle$ and $\left\langle\bar{E}_{\text {pot }}\right\rangle$ to be calculated. As the acoustic impedance is determined as a function of frequency, the calculation of $F_{\mathrm{ac}}$ is not limited to the resonant condition.

\subsubsection{Drag forces}

For low particle Reynold's number, $R e_{\mathrm{p}}<0.2$, the drag force $F_{\mathrm{D}}$ on a particle is calculated using Stokes drag, where $A$ is the area of the particle in a plane normal to the flow and the velocity $U_{0}$ the relative velocity between the fluid $U(x, y)$ and particle $u(x, y)$

$F_{\mathrm{D}}=\frac{1}{2} C_{\mathrm{D}} \rho_{\mathrm{f}} U_{0}^{2} A$

where $C_{\mathrm{D}}=24 / R e$ and $R e_{\mathrm{p}}=2 R \mu U_{0} / \rho_{\mathrm{f}}$.

Combining $u(x, y)$ and $U(x, y)$ with Eq. (3) and the expressions for $C_{\mathrm{D}}$ and $R e_{\mathrm{p}}$ gives:

$F_{\mathrm{D}}(x, y)=6 \mu \pi R(U(x, y)-u(x, y))$

To determine the drag force, the velocity profile across the depth of the main channel must be known. This can be determined numerically using CFD software but in this case, as laminar flow dominates, a parabolic profile describing flow between parallel plates [9] may be assumed,

$U_{x}=\frac{6 \bar{U}}{h^{2}}\left(h y-y^{2}\right)$

where $\bar{U}$ is the mean fluid velocity and $h$ the depth of the channel.

\subsubsection{Buoyancy}

The relative densities of the particle and fluid control the buoyancy force on a particle, where $F_{\mathrm{B}}$ opposes gravity:

$F_{\mathrm{B}}=\frac{4}{3} \pi R^{3} g\left(\rho_{\mathrm{f}}-\rho_{\mathrm{p}}\right)$

\subsection{Particle trajectories}

By summing the forces in both $x$ and $y$ directions a system of equations can be formed to give accelerations $\ddot{x}$ and $\ddot{y}$ for a particle of mass $m$. Note that both buoyancy and drag terms have $x$ and $y$ components, but the acoustic radiation force is assumed to be significant in the $y$ direction only (this allows for the option of inclining the cell)

$\ddot{x}=\frac{\sum F_{x}}{m}=\frac{F_{\mathrm{D}_{x}}+F_{\mathrm{B}_{x}}}{m}$

$\ddot{y}=\frac{\sum F_{y}}{m}=\frac{F_{\mathrm{ac}}+F_{\mathrm{D}_{y}}+F_{\mathrm{B}_{y}}}{m}$

This system of equations is solved in MATLAB using an ODE numerical solver function, where $F_{\mathrm{ac}}$ is extracted from the acoustic impedance transfer model implemented in MATLAB by Hill and Wood [7]. The ODE solver provides a series of particle coordinate locations and velocity components and therefore the trajectory of a particle, examples of which are shown in Fig. 2a. 


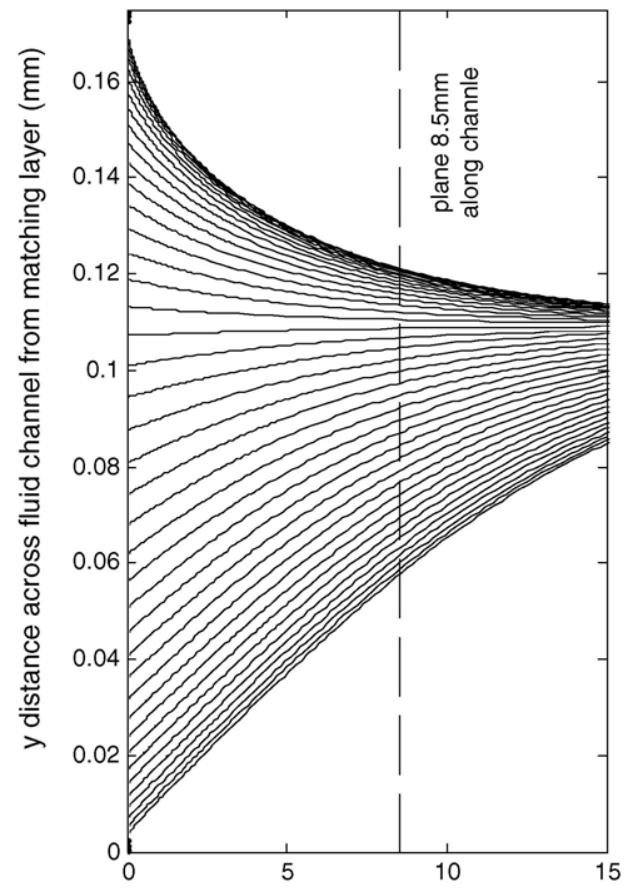

(a)

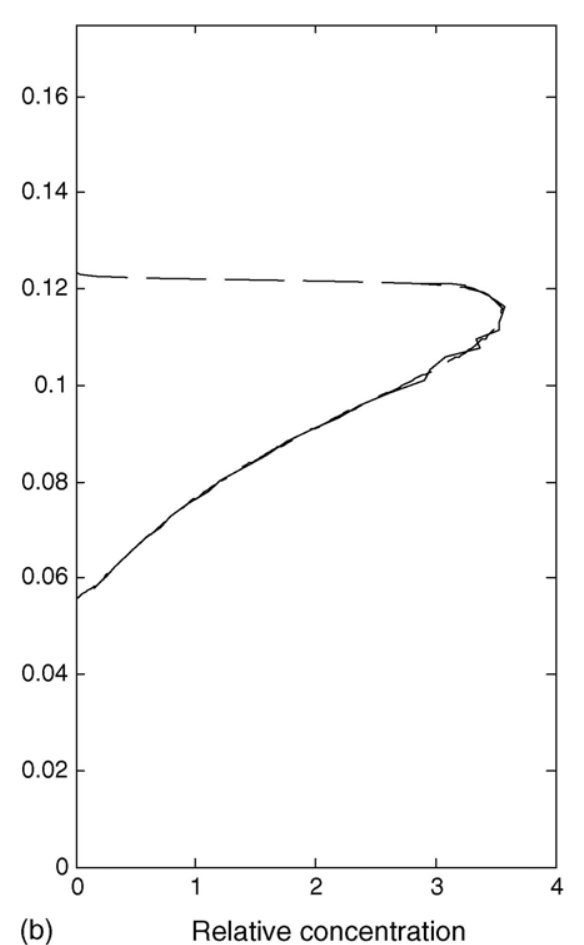

(b)

Fig. 2. (a) Convergence of particles towards nodal plane and (b) relative particle concentration across channel at a distance $8.5 \mathrm{~mm}$ along channel and acoustic field (relative concentration at inlet $=1$ ) and where $h=175 \mu \mathrm{m}$.

By modelling a series of particles at the channel inlet and using the resulting particle coordinates, it is possible to calculate the change in concentration across the channel [4]. The change in concentration is dependent on the change in lateral $(y)$ spacing between each particle. Based on the example given in Fig. 2a, at a distance $8.5 \mathrm{~mm}$ along the acoustic field (the edge of the acoustic field in a typical device) the particle trajectories illustrated result in the relative concentration distribution plotted in Fig. 2b.

Here, it is again important to consider the influence of the non-uniform flow profile; according to continuity, particle flow rate must remain constant therefore as a stream of particles moves into a plane of increased fluid velocity, the longitudinal $(x)$ spacing between particles must increase thus reducing the concentration seen at that point.

In the ultrasonic separator, two outlets split the flow and depending on the relative flow rates through these outlets the flow can be assumed to divide at a height $y=h N$. The particle flow rate passing through outlet 1 , for example, can be calculated using the concentration data described previously within the bounds of $0<y<h N$. Further, the concentration through the outlet is the particle flow rate divided by the fluid flow rate. A similar method within the bounds $h N<y<h$ can be used for outlet 2 or alternatively, by considering mass (particle) continuity. It is this method that is used to determine the predicted outlet concentration values presented in this paper.

\section{Experiment}

The experimental set-up consists of two peristaltic pumps, one feeding the inlet of the separator device and one drawing fluid through one of the two outlets. The device is isolated from the pulses produced by the pumps by using air filled syringes to act as dampers as shown in Fig. 3.

The transucer was driven via a fixed gain $(50 \mathrm{~dB}) \mathrm{RF}$ power amplifier by a signal generator at the resonant frequency of the chamber, previously determined to be a nominal $3.5 \mathrm{MHz}$. Fine adjustment was made by monitoring the transducer voltage with an oscilloscope and adjusting the frequency for a voltage minimum.

The flow rates through the outlets were chosen such that $Q_{1}<Q_{2}$. Therefore, operating in the half-wavelength mode with a particle stream formed along the centre of the separator channel, clear fluid can be extracted through outlet 1 and particle concentrate through outlet 2 .

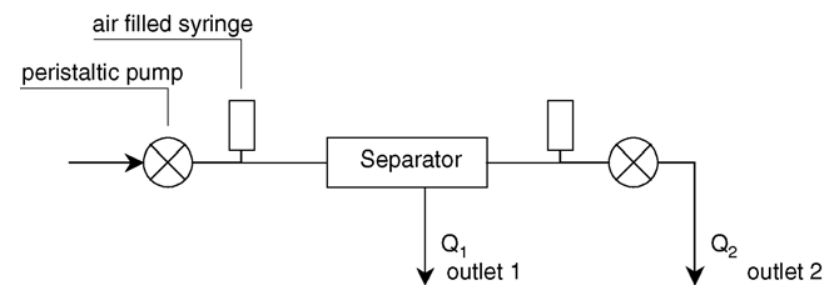

Fig. 3. Schematic of experiment. 


\subsection{Concentration measurement}

For each test, the fluid through each outlet was collected and its concentration measured using a turbidity sensor (Honeywell APMS-10GRCF) [5], previously calibrated for the particles under test.

\subsubsection{Sensor calibration}

This sensor offers the possibility of a low-cost, flowthrough turbidity measuring system. The sensor is manufactured by Honeywell (Part number APMS-10GRCF) [5] and is a microprocessor controlled system that measures turbidity, conductivity and temperature. It was originally designed for use in low cost, high volume applications, and so calibration to assess its suitability for this application was necessary. Readings are output in digital form and can be updated once every $1.3 \mathrm{~s}$. The turbidity measurement is achieved optically by using both a transmissive and a $90^{\circ}$ reflective sensor, allowing ratiometric readings. The sensor can be connected to a PC via an RS232 interface board. Custom software was written in Visual Basic to allow ratiometric measurements to be calculated from the raw data, and to be logged.

Measurement values vary with the materials in the fluid, and therefore the device needs to be calibrated for each type of fluid/particle mix. An absolute calibration was carried out using $1 \mu \mathrm{m}$ latex particles in water, comparing the sensor reading with the actual number of particles in the sample counted using a microscope and haemocytometer. Fig. 4 shows the sensor output against actual particle count for $1 \mu \mathrm{m}$ latex particles [6].

In addition, a calibration curve for percentage by weight was also established for the yeast particles used. This is shown in Fig. 5.

\section{Results}

Experimental and modelled results showing the influence of transducer voltage on both separation of $1 \mu \mathrm{m}$ latex particles and yeast cells are shown in Figs. 6 and 7, respectively.

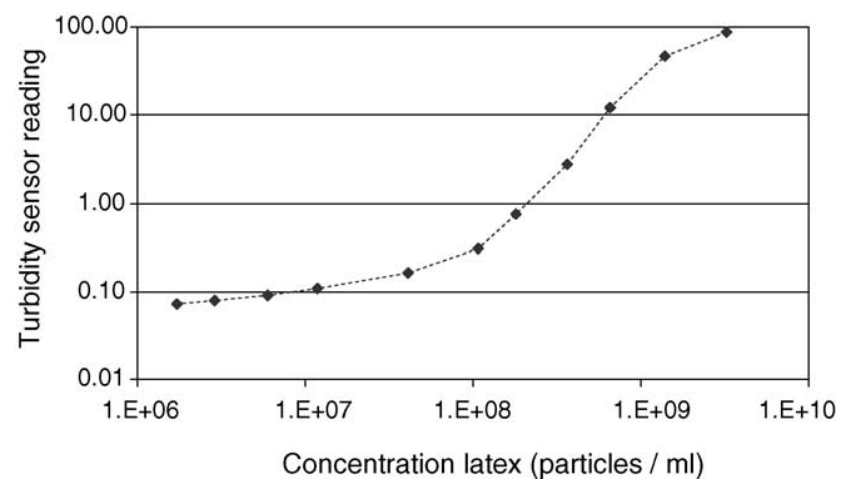

Fig. 4. One micron particle concentration against sensor output.

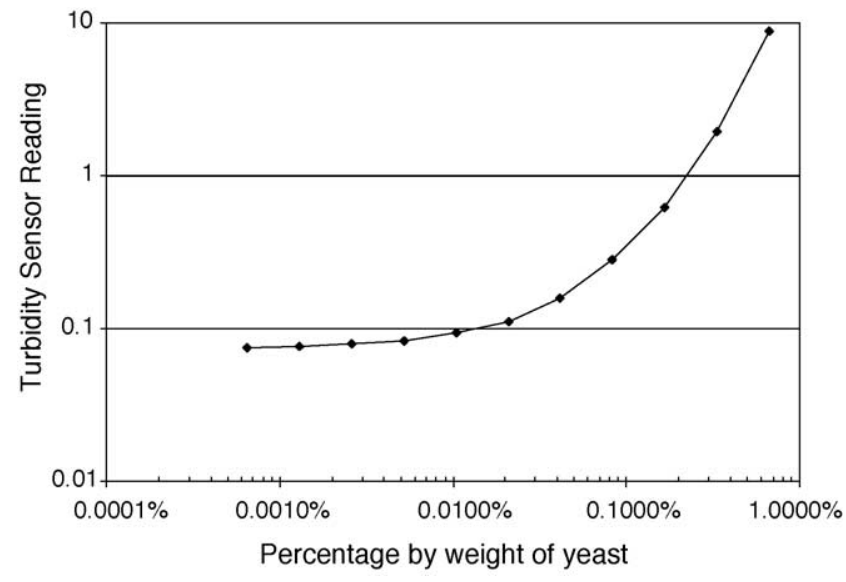

Fig. 5. Percentage of yeast by weight against sensor output.

In the experiments for yeast, a total flow rate $\left(Q_{1}+Q_{2}\right)$ of approximately $0.053 \mathrm{ml} / \mathrm{s}$ was maintained, $25 \%$ of which passed through outlet 1 . For the $1 \mu \mathrm{m}$ latex particles, these figures were $0.028 \mathrm{ml} / \mathrm{s}$ and $21 \%$, respectively.

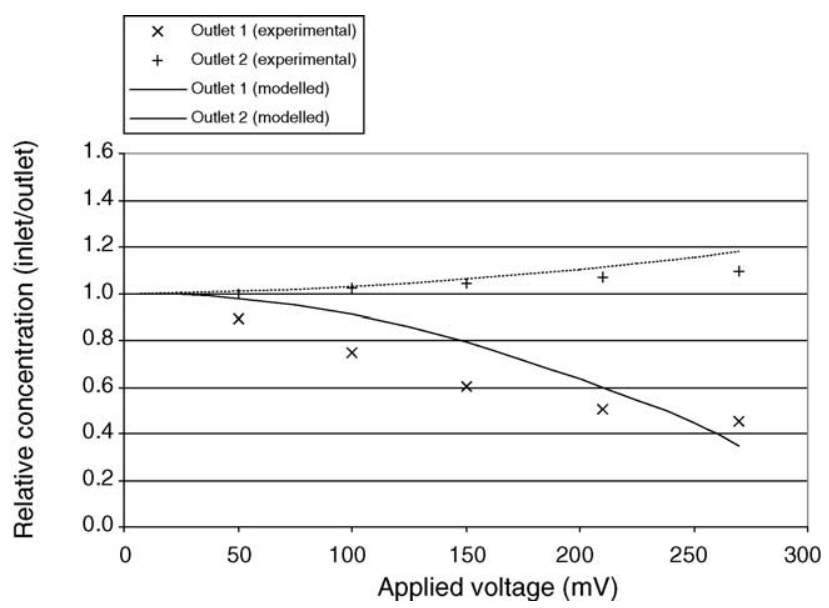

Fig. 6. Separation of $1 \mu \mathrm{m}$ latex particles based on an initial concentration of $0.016 \mathrm{wt} . \%$. Applied voltage is input voltage to amplifier.

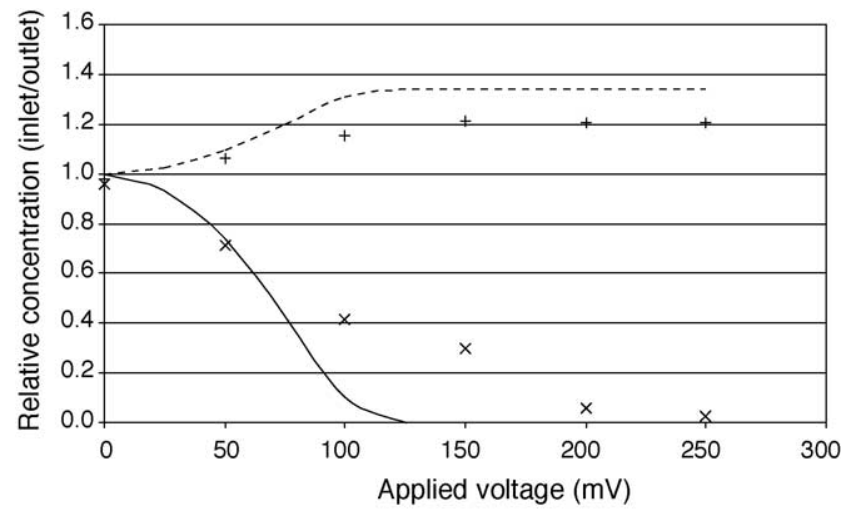

Fig. 7. Separation of yeast particles based on an initial concentration of 0.2 wt. $\%$. Applied voltage is input voltage to amplifier. 
Increasing transducer voltage increases the magnitude of the acoustic radiation force acting on suspended particles and in turn the rate at which they agglomerate on the nodal plane. Therefore, and as expected, the results show that increasing voltage causes more particles to pass through outlet 2 and decreases the concentration seen through outlet 1 .

The modelled results for the separation of $1 \mu \mathrm{m}$ particles (Fig. 6) compare well with the experiment results. Generally, the experimental results show slightly lower concentrations than modelled predictions, which can be attributed to the adhesion of particles on the walls within the fluid network surrounding the separator. At higher voltages, particles tend to clump together under higher acoustic forces, potentially disturbing the laminar flow required for successful separation and limiting the possible clearance through outlet 1 .

For the separation of yeast, the modelled results are more optimistic than the experimental results. The initial concentration of the yeast was high at $0.2 \%$ compared to that for the $1 \mu \mathrm{m}$ latex particles, where $0.015 \%$ was used. This high concentration may impede the motion of the yeast cells towards the nodal plane, indeed clustering was observed to be a greater problem with the yeast than with the latex particles. However, at higher voltages, separation is successful, producing a high degree of clarification. The degree of separation is influenced by flow rate as well as acoustic field strength, and a reduction of overall flow rate allows increased separation to occur. Further tests are required to investigate the influence of concentration and flow rates on the separation performance.

\section{Conclusions}

Previous work by the authors has investigated the acoustic fields within micro-engineered separators and this has allowed the influence of acoustic radiation forces on stationary particles to be successfully modelled. This work has extended this model to allow the path of particles flowing through the cell to be successfully predicted. The experimental work has verified the principles of the modelling, and this allows the combined model to be used to refine the performance of such separators before committing to production. In addition, the combined model can be used to investigate the feasibility of different configurations of the separator and is able to be easily tailored for specific particles, both in size and material properties. Operating parameters can then be predicted to give the required level of separation.

\section{Acknowledgements}

The authors wish to thank both the Engineering and Physical Research Council (EPSRC) and DSTL for their financial support under grant number GR/R13333/01. We also gratefully acknowledge the financial support given by Microfiltrex Ltd.

\section{References}

[1] M. Groschl, Ultrasonic separation of suspended particles, part I: fundamentals, Acustica 84 (3) (1998) 432-447.

[2] N.R. Harris, M. Hill, Y. Shen, R.J. Townsend, S. Beeby, N.M. White, A dual frequency, ultrasonic, micro-engineered particle manipulator, Ultrasonics 42 (2004) 139-144.

[3] N.R. Harris, M. Hill, S. Beeby, Y. Shen, N.M. White, J.J. Hawkes, W.T. Coakley, A silicon microfluidic ultrasonic separator, Sens. Actuators B 95 (2003) 425-434.

[4] R.J. Townsend, M. Hill, N.R. Harris, N.M. White, Modelling of particle paths passing through an ultrasonic standing wave, Ultrasonics 42 (2004) 319-324.

[5] Honeywell Sensing and Control Catalogue, Honeywell Inc., Freeport, IL, pp. 27-28.

[6] Polysciences Europe Gmbh, Eppelheim, Germany, www.polysciences.com.

[7] M. Hill, R.J.K. Wood, Modelling in the design of a flow-through ultrasonic separator, Ultrasonics 38 (2000) 662-665.

[8] M. Hill, Y.J. Shen, J.J. Hawkes, Modelling of layered resonators for ultrasonic separation, Ultrasonics 40 (2002) 385-392.

[9] F.M. White, Fluid Mechanics, 4th ed., McGraw-Hill, 1999.

\section{Biographies}

Nick Harris is a lecturer based in the Electronics and Computer Science Department at Southampton. He graduated in 1988 from the University of Bath and was awarded a PhD in 1997 from the University of Southampton, on the use of ultrasound for communication purposes. His research interests include thick-film sensors and surface acoustic wave devices, micro-engineering and sensor interfacing. $\mathrm{He}$ is a member of the IEE and a chartered engineer.

Rosemary Townsend graduated in 2000 from the University of Southampton with an MEng in mechanical engineering and is now studying for a $\mathrm{PhD}$ within the Electro-mechanical Research Group at Southampton. Her work involves the numerical simulation of particles under the influence of acoustic forces and other aspects of microfluidic device simulation. She is an associate member of the IMechE.

Martyn Hill is a reader in electromechanical systems in the School of Engineering Sciences, Southampton University. He graduated in 1985 from the Institute of Sound and Vibration Research, was appointed lecturer in 1990 and has research interests in acoustics (particularly ultrasonics) and transducers. His work has led to more than 80 publications, he is a chartered engineer, a member of the Institute of Measurement and Control, and a member of the Institute of Acoustics.

Steve Beeby graduated from the University of Portsmouth in 1992 with BEng (Hons) in mechanical engineering. He obtained a $\mathrm{PhD}$ from the University of Southampton in 1998. After his PhD, he obtained industrial funding for a follow up project to develop a resonant differential pressure sensor. He has since been awarded a EPSRC Advanced Research fellowship to continue his research into active thick film material development and their combination with micromachined devices. His other research interests, include energy harvesting for remote wireless sensor networks. $\mathrm{He}$ is also interested in human biometric systems. He has over 90 publications in the field. He is a co-author of a recently completed book entitled 'MEMS Mechanical Sensors' published by Artech House. He is a chartered engineer and chartered physicist. 
Neil M. White is a professor of intelligent sensor systems within the School of Electronics and Computer Science at the University of Southampton and also director of the Institute of Transducer Technology. He was awarded a PhD in 1988 for a thesis on the application of thick-film piezoresistors for load cells. He has published extensively in the area of thick-film sensors and intelligent instrumentation and is author or co-author of over 100 scientific publications. He is a fellow of the Institute of Physics, a chartered engineer and a senior member of the IEEE and has served of several committees in various professional bodies. 